\title{
Bicycle helmets and the law
}

\author{
Canadian legislation had minimal effect on serious head injuries
}

\author{
Ben Goldacre Wellcome research fellow in epidemiology ${ }^{1}$, David Spiegelhalter Winton professor \\ for the public understanding of risk ${ }^{2}$
}

${ }^{1}$ London School of Hygiene and Tropical Medicine, London WC1E 7HT, UK; ${ }^{2}$ University of Cambridge, Cambridge, UK

We have both spent a large part of our working lives discussing statistics and risk with the general public. We both dread questions about bicycle helmets. The arguments are often heated and personal; but they also illustrate some of the most fascinating challenges for epidemiology, risk communication, and evidence based policy.

With regard to the use of bicycle helmets, science broadly tries to answer two main questions. At a societal level, "what is the effect of a public health policy that requires or promotes helmets?" and at an individual level, "what is the effect of wearing a helmet?" Both questions are methodologically challenging and contentious.

The linked paper by Dennis and colleagues (doi:10.1136/bmj. f2674) investigates the policy question and concludes that the effect of Canadian helmet legislation on hospital admission for cycling head injuries "seems to have been minimal."1 Other ecological studies have come to different conclusions, ${ }^{2}$ but the current study has somewhat superior methodology—controlling for background trends and modelling head injuries as a proportion of all cycling injuries.

This finding of "no benefit" is superficially hard to reconcile with case-control studies, many of which have shown that people wearing helmets are less likely to have a head injury. ${ }^{3}$ Such findings suggest that, for individuals, helmets confer a benefit. These studies, however, are vulnerable to many methodological shortcomings. If the controls are cyclists presenting with other injuries in the emergency department, then analyses are conditional on having an accident and therefore assume that wearing a helmet does not change the overall accident risk. There are also confounding variables that are generally unmeasured and perhaps even unmeasurable. People who choose to wear bicycle helmets will probably be different from those who ride without a helmet: they may be more cautious, for example, and so less likely to have a serious head injury, regardless of their helmets.

People who are forced by legislation to wear a bicycle helmet, meanwhile, may be different again. Firstly, they may not wear the helmet correctly, seeking only to comply with the law and avoid a fine. Secondly, their behaviour may change as a consequence of wearing a helmet through "risk compensation," a phenomenon that has been documented in many fields. ${ }^{45}$ One study —albeit with a single author and subject—suggests that drivers give larger clearance to cyclists without a helmet. ${ }^{6}$

Even if helmets do have an effect on head injury rates, it would not necessarily follow that legislation would have public health benefits overall. This is because of "second round" effects, such as changes in cycling rates, which may affect individual and population health. Modelling studies have generally concluded that regular cyclists live longer because the health effects of cycling far outweigh the risk of crashes. ${ }^{7}$ This trade-off depends crucially, however, on the absolute risk of an accident: any true reduction in the relative risk of head injury will have a greater impact where crashes are more common, such as for children. ${ }^{8}$

The impact on all cause mortality, and on head injuries, may be even further complicated if such legislation has varying effects on different groups. For example, a recent study identified two broad subpopulations of cyclist: "one speed-happy group that cycle fast and have lots of cycle equipment including helmets, and one traditional kind of cyclist without much equipment, cycling slowly." The study concluded that compulsory cycle helmet legislation may selectively reduce cycling in the second group. ${ }^{9}$ There are even more complex second round effects if each individual cyclist's safety is improved by increased cyclist density through "safety in numbers," a phenomenon known as Smeed's law. ${ }^{10}$ Statistical models for the overall impact of helmet habits are therefore inevitably complex and based on speculative assumptions. ${ }^{11}$ This complexity seems at odds with the current official BMA policy, which confidently calls for compulsory helmet legislation.

Standing over all this methodological complexity is a layer of politics, culture, and psychology. Supporters of helmets often tell vivid stories about someone they knew, or heard of, who was apparently saved from severe head injury by a helmet. Risks and benefits may be exaggerated or discounted depending on the emotional response to the idea of a helmet. ${ }^{12}$ For others, this is an explicitly political matter, where an emphasis on helmets reflects a seductively individualistic approach to risk 
management (or even "victim blaming") while the real gains lie elsewhere. It is certainly true that in many countries, such as Denmark and the Netherlands, cyclists have low injury rates, even though rates of cycling are high and almost no cyclists wear helmets. This seems to be achieved through interventions such as good infrastructure; stronger legislation to protect cyclists; and a culture of cycling as a popular, routine, non-sporty, non-risky behaviour.

In any case, the current uncertainty about any benefit from helmet wearing or promotion is unlikely to be substantially reduced by further research. Equally, we can be certain that helmets will continue to be debated, and at length. The enduring popularity of helmets as a proposed major intervention for increased road safety may therefore lie not with their direct benefits-which seem too modest to capture compared with other strategies - but more with the cultural, psychological, and political aspects of popular debate around risk.

Competing interests: We have read and understood the BMJ Group policy on declaration of interests and declare the following interests: None.

Provenance and peer review: Commissioned; not externally peer reviewed.
1 Dennis J, Ramsay T, Turgeon AF, Zarychanski R. Helmet legislation and admissions to hospital for cycling related head injuries in Canadian provinces and territories: interrupted time series analysis. BMJ 2013;346:f2674.

2 Macpherson A, Spinks A. Bicycle helmet legislation for the uptake of helmet use and prevention of head injuries. Cochrane Database Syst Rev 2008;3:CD005401.

3 Thompson DC, Rivara F, Thompson R. Helmets for preventing head and facial injuries in bicyclists. Cochrane Database Syst Rev 2000;2:CD001855. http://summaries.cochrane. org/CD001855/wearing-a-helmet-dramatically-reduces-the-risk-of-head-and-facial-injuriesfor-bicyclists-involved-in-a-crash-even-if-it-involves-a-motor-vehicle.

4 Phillips RO, Fyhri A, Sagberg F. Risk compensation and bicycle helmets. Risk Anal 2011;31:1187-95.

5 Adams J. Risk. Taylor \& Francis, 2002.

6 Walker I. Drivers overtaking bicyclists: objective data on the effects of riding position, helmet use, vehicle type and apparent gender. Accid Anal Prev 2007:39:417-25.

7 WHO. Quantifying the health benefits of cycling and walking. www.euro.who.int/en/whatwe-do/health-topics/disease-prevention/physical-activity/activities/quantifying-the-healthbenefits-of-cycling-and-walking.

8 De Jong P. The health impact of mandatory bicycle helmet laws. Risk Anal 2012;32:782-90.

9 Fyhri A, Bjørnskau T, Backer-Grøndahl A. Bicycle helmets-a case of risk compensation? Transp Res Part F Traffic Psychol Behav 2012;15:612-24.

10 Robinson D. Safety in numbers in Australia: more walkers and bicyclists, safer walking and bicycling. Health Promot J Austr 2005;16:47-51.

11 Newbold SC. Examining the health-risk tradeoffs of mandatory bicycle helmet laws. Risk Anal 2012;32:791-8.

12 Slovic P, Peters E, Finucane M, MacGregor D. Affect, risk and decision making. Health Psychol 2005;24:S35-40.

Cite this as: BMJ 2013;346:f3817

(c) BMJ Publishing Group Ltd 2013 